\title{
ATRIBUTOS QUÍMICOS E PRODUÇÃO DE MILHO EM UM LATOSSOLO VERMELHO EUTROFÉRRICO TRATADO COM LODO DE ESGOTO(1)
}

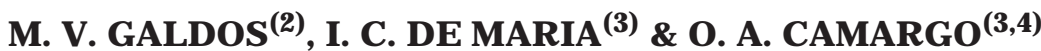

\begin{abstract}
RESUMO
A utilização de lodo de esgoto como fertilizante orgânico ou condicionador do solo, tem-se tornado cada vez mais atraente, pelos altos custos e impactos ambientais relacionados com os demais métodos de disposição, pela presença de nutrientes vegetais e matéria orgânica no lodo e pela necessidade de redução de custos na agricultura. No entanto, em sua composição, pode conter metais pesados, microrganismos patogênicos e compostos orgânicos tóxicos. Visando estudar os efeitos da aplicação de lodo de esgoto em áreas agrícolas, foi realizado um experimento, com duração de dois anos agrícolas, para determinar alterações em propriedades químicas do solo, princi palmente sobre os teores de $\mathrm{P}, \mathrm{Cu}, \mathrm{Ni}$ e Zn em um Latossolo Vermelho eutroférrico, de textura argilosa, cultivado com milho. $O$ experimento consistiu na aplicação de lodo de esgoto em duas doses comparadas a uma testemunha com adubação química. Para análise de rotina das propriedades químicas e do teor de $\mathrm{Ni}$ do solo, foram coletadas amostras nas profundidades de 0-0,05; 0,05-0,10 e 0,10-0,20 $\mathrm{m}$ após a collheita do milho no primeiro e no segundo ano. Foi feita, também, uma extração seqüencial de $P$ em amostras da camada de 0-0,05 m, na seguinte ordem: $\mathrm{CaCl}_{2}$ (P biologicamente mais disponível); $\mathrm{NaHCO}_{3}$ ( $\mathrm{P}$ disponível); $\mathrm{NaOH}$ ( $\mathrm{P}$ ligado aos óxidos de $\mathrm{Fe}$ e Al); $\mathrm{HCl}$ (P ligado a $\mathrm{Ca}$ ) e digestão nítrico-perclórica ( $\mathrm{P}$ residual). A produção do milho foi maior nos tratamentos com aplicação do lodo. Os teores disponíveis de $\mathbf{P}$ no solo onde foi aplicado lodo foram semelhantes aos do tratamento sem lodo e com adubo químico. E ntretanto, a aplicação do lodo aumentou as frações lábeis e moderadamente lábeis do P na camada superficial. Os dados indicaram, por outro lado, aumento dos teores de $\mathrm{Cu}, \mathrm{Ni}$ e $\mathrm{Zn}$ no solo e de $\mathrm{Zn}$ na planta. Dessa forma, faz-se necessário constante monitoramento do solo onde o lodo de esgoto é aplicado para o controle adequado dos teores de metais.
\end{abstract}

Termos de indexação: biossólido, poluição, fósforo, zinco, níquel e cobre.

\footnotetext{
(1) Parte da Tese de Mestrado do primeiro autor, Curso de Pós-Graduação em Agricultura Tropical e Subtropical, Instituto Agronômico - IAC. Projeto financiado com recursos da FAPESP. Recebido para publicação em junho de 2003 e aprovado em maio de 2004.

(2) Mestrando do Instituto Agronômico - IAC, Curso de Pós-Graduação em Agricultura Tropical e Subtropical. Caixa Postal 28, CEP 13001-970 Campinas (SP). E-mail: mvgaldos@hotmail.com

(3) Pesquisador Científico, IAC. E-mail: icdmaria@iac.sp.gov.br; ocamargo@iac.sp.gov.br

(4) Bolsista do CNPq.
} 


\title{
SUMMARY: SOIL CHEMICAL PROPERTIES AND CORN PRODUCTION IN A SEWAGE SLUDGE-AMENDED SOIL
}

\begin{abstract}
The application of sewage sludge into agricultural soils, as organic fertilizer or soil conditioner, is becoming an increasingly moreattractivealternative It is occurring because of the high costs and environmental impacts caused by other sludge disposal methods, besides the presence of plant nutrients and organic matter in the sludge and the need to reduce costs in agriculture. However, sewagesludge can contain heavy metals, pathogenic microorganisms, and toxic organic compounds. In order to study the impact of sewage sludge application in agricultural areas, a two-year experiment was implemented to determinethechanges in soil chemical properties, mainly in the P, $\mathrm{Cu}, \mathrm{Ni}$ and $\mathrm{Zn}$ contents, in an eutroferric clayey Red Latosol (Rhodic E utrustox) cropped with corn. Theexperiment consisted of two application rates of sewagesludgeand a control with chemical fertilization. Soil samples were collected at the $0-0.05 ; 0.05-0.10$, and $0.10-0.20 \mathrm{~m}$ depths after the corn harvest in both years for a routine chemical analysis and the Ni content. Additionally, a sequential $\mathrm{P}$ extraction for samples of the 0 to $0.05 \mathrm{~m}$ layer was carried out in thefol lowing order: $\mathrm{CaCl}_{2}$ ( $\mathrm{P}$ biologically most available); $\mathrm{NaHCO}_{3}$ (availableP); $\mathrm{NaOH}$ ( $\mathrm{P}$ adsorbed to the Feand $\mathrm{Al}$ oxides); $\mathrm{HCl}$ (P adsorbed to $\mathrm{Ca}$ ) and nitric-perchloric digestion (residual $\mathrm{P}$ ). Corn yiel d was higher in thetreatments with sludgeapplication. TheavailableP content in soils that recei ved sludge application was similar to those of the treatment without sludge and with chemical fertilizer. However, sludgeapplication increased thelabileand moderately labile P fractions in the surfacesoil layer. The data suggested, however, increased $\mathrm{Cu}, \mathrm{Ni}$, and $\mathrm{Zn}$ concentration in soil and $\mathrm{Zn}$ concentration in plants. Thus, a constant monitoring of soils that receive application of sewage sludge is required for an adequate control of the metal levels.
\end{abstract}

Index terms: bi osolids, pollution, phosphorus, zinc, nickel and copper.

\section{INTRODUÇÃO}

A aplicação de lodo de esgoto em solos agrícolas, como fertilizante orgânico ou condicionador do solo, deve crescer substancialmente nos próximos anos no Brasil, seguindo uma tendência mundial e acompanhandoa demanda gerada por um acentuado crescimento no volume de esgoto tratado no País (Tsutiya, 2001).

Há, no momento, por pressão da sociedade civil e maior val orização dos recursos hídricos pela opi nião pública, um acentuado crescimento no volume de esgoto tratado no País. N ovas ETEs (Estações de Tratamento deE sgoto) estão surgindo eas existentes estão expandindo sua capacidade. O tratamento de esgotos gera, além de águas residuais com baixa carga poluidora, um resíduo chamado de lodo de esgoto ou biossólido, composto por matéria orgânica, nutrientes e alguns el ementos potencialmente tóxicos. A disposição adequada deste resíduo é um dos principais desafios a ser enfrentado pelos gestores ambientais.

Os métodos de disposição mais comuns para o lodo são: incineração, aterro, disposição no oceano, recuper ação de terrenos de mineração, digestão em lagoas e uso agrícola (Harris-Pierce et al., 1995). Atualmente, diversos usos alternativos têm sido desenvolvidos, como: agregados leves para construção civil, fabricação de tijolos e cerâmicas, fonte de energia para produção de cimento e conversão do lodo em óleo combustível (Tsutiya, 2001). Além disso, a aplicação deste resíduo em terras agrícolas tem-se tornado cada vez mais atraente, pelos altos custos e impactos ambientais relacionados com os demais métodos de disposição, pela presença de nutrientes e matéria orgânica no lodo e pela necessidade de redução de custos na agricultura.

O lodo de esgoto, que varia em sua composição de acordo com a origem e método de tratamento, tem si do usado como fertilizante orgânico, por conter alguns elementos essenciais às plantas, tais como: N, P, Ca, Mg, S emicronutrientes (M el o et al., 1994; Polglase\& Myers, 1996; Berton et al., 1997; Silva et al., 2002). O lodo também tem sido val orizado como condicionador do solo, aumentando a capacidade de retenção de água, a porosidade e a estabilidade dos agregados, por meio da adição de matéria orgânica ao solo (Elliot, 1986; J orge et al. 1991; Wilson et al., 1996).

No entanto, a utilização do lodo de esgoto na agricultura não é isenta de problemas. Em sua composição, ol odo pode conter metais pesados, como $\mathrm{Cd}, \mathrm{Cr}, \mathrm{Cu}, \mathrm{Ni}, \mathrm{Pb}, \mathrm{Fe}, \mathrm{Co}, \mathrm{Mn}, \mathrm{Mo}, \mathrm{Hg}$, Sn e Zn, 
microrganismos patogênicos e compostos orgânicos tóxicos, representando, portanto, riscos diretos ou indiretos à saúde humana e animal (Elliot, 1986, Gibbs et al., 1997, Tan, 2000). Além dos metais pesados, o lodo contém altos teores de $\mathrm{N}$ e $\mathrm{P}$. A legislação atual referente à disposição de lodo de esgoto no estado de São Paulo (Norma P 4230, CETESB, 1999) estabelece limites quanto às quantidades de lodo que podem ser aplicadas no solo, baseando-se, principalmente, nos teores de metais pesados. Uma vez que a dose aplicada em áreas agrícolas é calculada de acordo com os teores de $\mathrm{N}$ no lodo com a necessidade deste el emento pela cultura, pode ocorrer um acúmulo de $\mathrm{P}$ e metais pesados no solo.

Para estudar os efeitos da aplicação de lodo de esgoto em áreas agrícolas, foi realizado um ensaio para determinar alterações em atributos químicos do solo, especialmente nos teores disponíveis de $\mathrm{P}$, $\mathrm{Cu}, \mathrm{Ni}$ e Zn em solo cultivado com milho.

\section{MATERIAL E MÉTODOS}

O experimento, com duração de dois anos agrícolas, foi instalado em dezembro de 2001 no Centro Experimental do Instituto Agronômico em Campinas, que se localiza na latitude 22 ㅇ ' Sul e longitude 47 1 ' Oeste, com altitude de 600 a 720 m. O clima da região, segundo a classificação de Köppen (Critchfield, 1960), é do tipo Cwa, definido como tropical úmido com estação chuvosa distinta noverão e seco no inverno, com temperatura média anual de $20,5^{\circ} \mathrm{C}$. A precipitação média anual éde $1.400 \mathrm{~mm}$, com a distribuição de chuvas de $76 \%$ de outubro a março (dados coletados por trinta anos na estação pluviométrica instalada próxima às parcelas).

O solo da área experimental é um Latossolo Vermelho eutroférrico, de textura argilosa, classificado como Typic Haplortox na Classificação do USDA e como Rhodic Ferrasol na classificação da FAO. O quadro 1 apresenta a caracterização inicial dos atributos químicos do solo, nas profundidades de 0-0,05; 0,05-0,10 e 0,10-0,20 m. Posteriormente, em 3 de outubro de 2001, a área recebeu calagem na dose $4 \mathrm{Mg} \mathrm{ha}^{-1}$.
O experimento consistiu na aplicação de lodo de esgoto em duas doses (L1 e L2) para comparação com uma testemunha, sem aplicação do lodo (L0). $O$ del ineamento experimental, em faixas com quatro repetições, utilizou doze parcelas com área útil de $100 \mathrm{~m}^{2}(4 \times 25 \mathrm{~m})$ em declive uniforme de $10 \%$. Cada parcela foi dividida em três subparcelas (terço superior, médio e inferior), aumentando o número de graus de liberdade para a análise estatística.

Utilizou-se lodo de esgoto (LE) proveniente da Estação deTratamento de Esgoto (ETE) deJ undiaí, SP. O processo de tratamento de esgoto do qual resulta o lodo é composto por lagoas aeradas de mistura completa seguida de lagoas de decantação. O LE é, em seguida, desaguado por centrífugas e ocorrea adição de poliel etról itos, sendo condicionado física e sanitariamente por 60 a 90 dias, sofrendo revolvimento mecânico em um pátio coberto.

As doses de lodo foram definidas com base em sua anál ise química e na necessi dade de $\mathrm{N}$ da cultura (CETESB, 1999), sendo a dose testemunha L0, sem aplicação de lodo e com adubação química, a dose $L 1$ aquela recomendada e a dose $L 2$ o dobro da recomendada. A primeira aplicação foi feita em 18/ 12/2001, sendo L $0, L 1$ e L 2, respectivamente $0 ; 10,8$ e 21,6 Mg de massa seca de lodo por hectare. A segunda aplicação foi feita em 18/11/2002. O cál culo da dose aplicada no segundo ano incluiu a taxa de $10 \%$ de decaimento do lodo (efeito residual) aplicado no primeiro ano e, assim, as doses $L 0, L 1$ e $L 2$, no segundo ano, foram, respectivamente: 0; 10,2 e $20,5 \mathrm{Mg}$ de massa seca de lodo por hectare. O lodo foi incorporado ao solo a uma profundidade de 0,10 m, com enxadão.

O milho, variedade IAC 21, foi semeado em contorno, com espaçamento de 0,90 m entre linhas e cinco plantas por metrolinear. As semeaduras foram realizadas em 19 de dezembro de 2001 e em 19 de novembro de 2002. Na dose L 0 , a cultura recebeu adubação NPK no plantio, sendo $4,8 \mathrm{~kg} \mathrm{ha}^{-1}$ de N, $21 \mathrm{~kg} \mathrm{ha}^{-1}$ de $\mathrm{P}$ e 14,5 kg ha-1 de K. Em virtude da baixa concentração de $\mathrm{K}$ no lodo de esgoto, foi aplicado $\mathrm{KCl}$ na mesma dosagem da fórmula de NPK, ou seja, 14,5 kg ha-1 deK nas parcelas das doses $\mathrm{L} 1 \mathrm{e}$ L2. Foi feita adubação de cobertura com uréia, aplicando-se $74 \mathrm{~kg} \mathrm{ha}^{-1}$ deN em 29 dejaneiro de 2002

Quadro 1. Valores médios dos atributos químicos do solo antes da instalação do experimento nas profundidades de 0-0,05, 0,05-0,10 e 0,10-0,20 m

\begin{tabular}{|c|c|c|c|c|c|c|c|c|c|c|c|c|c|c|c|}
\hline Profundidade & pH $\left(\mathrm{CaCl}_{2}\right)$ & $\mathbf{H}+\mathbf{A l}$ & $\mathbf{s}$ & СТC & $\mathbf{v}$ & $\mathbf{P}$ & $\mathbf{K}$ & $\mathrm{Ca}$ & Mg & MO & B & $\mathrm{Cu}$ & $\mathbf{F e}$ & Mn & Zn \\
\hline $\mathrm{m}$ & & \multicolumn{3}{|c|}{$\mathrm{mmol}_{\mathrm{c}} \mathrm{dm}^{-3}$} & $\%$ & $\mathrm{mg} \mathrm{dm}^{-3}$ & \multicolumn{3}{|c|}{$-\mathrm{mmol}_{\mathrm{c}} \mathrm{dm}^{-3}$} & $\mathrm{~g} \mathrm{dm}^{-3}$ & 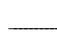 & 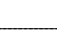 & $\mathrm{gg} \mathrm{dm}$ & 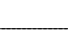 & $\underline{-}$ \\
\hline $0-0,05$ & 4,6 & 48,8 & 16,6 & 65,3 & 25,7 & 36,1 & 3,9 & 9,3 & 3,3 & 26,2 & 0,3 & 8,1 & 23,8 & 62,5 & 1,5 \\
\hline $0,05-0,10$ & 4,6 & 46,2 & 15,9 & 62,0 & 26,2 & 19,4 & 2,2 & 10,4 & 3,3 & 23,4 & 0,2 & 7,7 & 20,8 & 56,8 & 0,7 \\
\hline $0,10-0,20$ & 4,7 & 41,6 & 17,4 & 59,0 & 30,3 & 12,6 & 1,8 & 12,2 & 3,4 & 12,6 & 0,2 & 7,4 & 19,4 & 50,7 & 0,5 \\
\hline
\end{tabular}


na dose L0 no, primeiro ano, e $81 \mathrm{~kg} \mathrm{ha}^{-1}$ de $\mathrm{N}$ em 8 de janeiro de 2003 nas três doses, no segundo ano.

Antes de cada aplicação, foram coletadas três amostras do lodo de esgoto para avaliação dos seguintes atributos químicos: (a) $\mathrm{P}, \mathrm{B}, \mathrm{As}, \mathrm{Cd}, \mathrm{Pb}$, $\mathrm{Cu}, \mathrm{Cr}, \mathrm{Hg}, \mathrm{Mo}, \mathrm{Ni}, \mathrm{Se}, \mathrm{Zn}, \mathrm{S}, \mathrm{Mn}, \mathrm{Fe}, \mathrm{Mg}, \mathrm{Al}, \mathrm{Ca}$, $\mathrm{Na}$ e K, pelo método EPA 3051 (Abreu et al., 2001), com determinação feita por espectrofotometria de plasma (ICP-OES); (b) carbono orgânico pel o método do bloco digestor, com determinação por titulometria; (c) sólidos voláteis: foram pesados $2,5 \mathrm{~g}$ da amostra seca a $60^{\circ} \mathrm{C}$, e colocados na mufla a $500{ }^{\circ} \mathrm{C}$ por $4 \mathrm{~h}$. Após este período, a amostra foi pesada novamente; (d) $\mathrm{N} \mathrm{Kjeldahl} \mathrm{(total)} \mathrm{e} \mathrm{NH}_{4}{ }^{+} \mathrm{e}$ $\mathrm{NO}_{3}{ }^{-}$pelo método descrito para análise de solo por Cantarella \& Trivelin (2001).

Foram coletadas amostras de solo nas profundidades de 0-0,05; 0,05-0,10 e 0,10-0,20 m para avaliação dos teores disponíveis dos el ementos após a colheita no primeiro ano, em 11/07/02 e no segundo ano, em 16/05/03. Em todas as amostragens, foram col etadas cinco subamostras por subparcela para formar uma amostra composta.

As amostras de solo foram preparadas e analisadas de acordo com a rotina analítica do Instituto Agronômico de Campinas (Raij et al., 2001), determinando-se: $\mathrm{pH}$ em $\mathrm{CaCl}_{2}$ 0,01 mol L-1; $\mathrm{P}$ por extração com resina trocadora deíon e determinado pelo método do vanadato-molibdato; matéria orgânica determinada pel o método col orimétrico do dicromato de Na. A soma debases (S), a percentagem de saturação por bases (V \%) e a capacidade de troca de cátions (CTC potencial) foram cal culadas a partir dos val ores de $\mathrm{K}, \mathrm{Ca}, \mathrm{Mg}$ (método da resina) e H + Al (método da solução tampão SMP); Zn, Ni, Cu, Fe e $\mathrm{Mn}$ extraídos pelo método do DTPA pH 7,3 e determinação por ICP-OES.

Para avaliar o efeito da aplicação de lodo na distribuição de $\mathrm{P}$ no solo, fracionaram-se amostras de solo na profundidade de $0-0,05 \mathrm{~m}$ no primeiro ano do experimento. O fracionamento foi feito de acordo com Hedley et al . (1982), modificado por Sui et al. (1999), na seguinte ordem: $\mathrm{CaCl}_{2}(\mathrm{P}$ biologicamente mais disponível); $\mathrm{NaHCO}_{3}$ (P disponível); $\mathrm{NaOH}$ (P ligado aos óxidos de $\mathrm{Fe}$ e $\mathrm{Al}$ ); $\mathrm{HCl}$ (P ligadoa $\mathrm{Ca}$ ) e digestão nítrico-perclórica ( $P$ residual).

Para efetuar a análise química das fol has, foram col etadas fol has da base da espiga de cinco plantas por subparcela no primeiro ano e dez por subparcela no segundo ano. A coleta foi feita na fase de pendoamento (50\% das plantas pendoadas), tendo sido reservado oterço central da fol ha, sem a nervura central. As amostras de fol has foram lavadas em uma solução detergente $(0,1 \% \mathrm{v} / \mathrm{v})$, enxaguadas em água destilada até à remoção do detergente e enxaguadas em água deionizada. Após a lavagem, as amostras foram colocadas em sacos de papel e secas a $65 \%$ em estufa de ventilação forçada, até o peso constante. Depois deseco, o material foi moído em moinho tipo Wiley, com câmara de aço inoxidável, com peneira de $1 \mathrm{~mm}$ de abertura (Bataglia et al., 1983b). O extrato de todas as amostras de fol has foi preparado por via seca (incineração de $1,00 \mathrm{~g}$ de material em mufla por $3 \mathrm{~h}$ a $500^{\circ} \mathrm{C}$ e dissolução da cinza em $\left.\mathrm{HCl} 6 \mathrm{~mol} \mathrm{~L}^{-1}\right)$. A determinação de $\mathrm{P}, \mathrm{Cu}$, $\mathrm{Ni}$ e $\mathrm{Zn}$ foi feita por espectrofotometria de plasma (ICP-OES).

A avaliação da produção foi feita por amostragem, col etando-se as plantas das duas linhas centrais de cada subparcela $(4 \times 1,8 \mathrm{~m})$, para determinação da massa de grãos a $13 \%$ de umi dade e massa seca da parte aérea (colmo, fol ha, sabugo e palha) a $65^{\circ} \mathrm{C}$ em estufa de ventilação forçada.

Os resultados obti dos foram submetidos à anál ise de variância com aplicação do teste $\mathrm{F}$ a $5 \%$. As médias dos tratamentos foram comparadas por meio da aplicação do teste " $t$ " para 0,05 de significância global.

\section{RESULTADOS E DISCUSSÃO}

\section{Características do lodo de esgoto}

Os resultados analíticos do lodo de esgoto (Quadro 2) são comparados aos limites determinados pela Norma P 4230 da CETESB. Os teores de metais pesados no lodo de esgoto (LE) utilizado neste experimento são bastante inferiores aos limites estabel ecidos, adequando-se ao uso agrícola quanto aos atributos químicos.

Em relação à quantidade de metais aplicada anualmente, as duas doses de LE utilizadas no experimento não ultrapassam os limites estabelecidos na norma P 4230. Os limites cumulativos descritos nesta mesma norma levariam $49,95,244,311$ e 465 anos para atingir os limites no sol o de Pb, Zn, Cu, Cd e Ni com aplicações anuais, mantendo-se constantes os teores no lodo aplicados no primeiroano. É importantefrisar queas medidas da Norma P 4230 são baseadas em legislação americana, cujos critérios foram desenvolvidos em condições edafocl imáticas diferentes das brasileiras. O estabelecimento de limites cumulativos no solo também pode ser questionado, uma vez que há poucos estudos de longo prazo que levem em conta as interações dos metais com o meio ambiente ao serem aplicados no solo.

\section{Teores de elementos químicos no solo}

No primeiro ano, a aplicação no sol o resultou em aumento nos teores de $\mathrm{P}$, passando de médio para alto na profundidade de $0-0,05 \mathrm{~m}$, e de baixo para médio de 0,05-0,10 m (Quadro 3). Não houve efeito da aplicação delodo nos teores de Cu e Ni, mas todos 
Quadro 2. Composi ção do lodo de esgoto utilizado no primeiro (2001/02) e no segundo (2002/03) ano do experimento e valores de concentração máxima permitida (CMP) pela legislação

\begin{tabular}{|c|c|c|c|}
\hline \multirow{2}{*}{ Característica $^{(1)}$} & \multicolumn{2}{|c|}{ Ano } & \multirow[b]{2}{*}{$\mathbf{C M P}^{(3)}$} \\
\hline & 2001 & 2002 & \\
\hline Fósforo $\left(\mathrm{g} \mathrm{kg}^{-1}\right)$ & 6,6 & 7,2 & \\
\hline Potássio $\left(\mathrm{g} \mathrm{kg}^{-1}\right)$ & 1,5 & 1,0 & \\
\hline Sódio $\left(\mathrm{g} \mathrm{kg}^{-1}\right)$ & 0,9 & 1,7 & \\
\hline Cromo total $\left(\mathrm{mg} \mathrm{kg}^{-1}\right)$ & 149,3 & 188,5 & \\
\hline Arsênio $\left(\mathrm{mg} \mathrm{kg}^{-1}\right)$ & $<0,1$ & $<0,01$ & 75 \\
\hline Cádmio (mg kg $\left.{ }^{-1}\right)$ & 5,8 & 6,6 & 85 \\
\hline Chumbo $\left(\mathrm{mg} \mathrm{kg}^{-1}\right)$ & 283,1 & 206,6 & 840 \\
\hline Cobre $\left(\mathrm{mg} \mathrm{kg}^{-1}\right)$ & 284,1 & 864,8 & 4.300 \\
\hline Mercúrio (mg kg $\left.{ }^{-1}\right)$ & $<0,1$ & $<0,01$ & 57 \\
\hline Molibdênio ( $\mathrm{mg} \mathrm{kg}^{-1}$ ) & $<0,1$ & $<0, .01$ & 75 \\
\hline Níquel $\left(\mathrm{mg} \mathrm{kg}^{-1}\right)$ & 41,8 & 35,5 & 420 \\
\hline Selênio $\left(\mathrm{m} \mathrm{kg}^{-1}\right)$ & $<0,1$ & $<0,01$ & 100 \\
\hline Zinco $\left(\mathrm{mg} \mathrm{kg}^{-1}\right)$ & $1.364,8$ & $1.738,1$ & 7.500 \\
\hline Boro $\left(\mathrm{mg} \mathrm{kg}^{-1}\right)$ & 12,3 & 11,7 & \\
\hline Carbono orgânico $\left(\mathrm{g} \mathrm{kg}^{-1}\right)$ & 325,4 & 298,5 & \\
\hline $\mathrm{pH}\left(\mathrm{CaCl}_{2}\right)$ & 6,6 & 5,5 & \\
\hline Umidade $(\%)$ & 68,2 & 65,7 & \\
\hline Sólidos Voláteis (\%) & 54,8 & 57,3 & \\
\hline Nitrogênio Kjeldahl $\left(\mathrm{g} \mathrm{kg}^{-1}\right)$ & 28,3 & 27,0 & \\
\hline Nitrogênio amoniacal $\left(\mathrm{mg} \mathrm{kg}^{-1}\right)^{(2)}$ & 577,4 & 438,2 & \\
\hline Nitrogênio nitrato/nitrito $\left(\mathrm{mg} \mathrm{kg}^{-1}\right)^{(2)}$ & 37,2 & 138,7 & \\
\hline Enxofre $\left(\mathrm{g} \mathrm{kg}^{-1}\right)$ & 26,1 & 26,8 & \\
\hline Manganês $\left(\mathrm{mg} \mathrm{kg}^{-1}\right)$ & 676,8 & 693,3 & \\
\hline Ferro $\left(\mathrm{mg} \mathrm{kg}^{-1}\right)$ & 26,0 & 24,1 & \\
\hline Magnésio $\left(\mathrm{g} \mathrm{kg}^{-1}\right)$ & 2,1 & 1,7 & \\
\hline Alumínio $\left(\mathrm{mg} \mathrm{kg}^{-1}\right)$ & 16,4 & 18,8 & \\
\hline Cálcio $\left(\mathrm{g} \mathrm{kg}^{-1}\right)$ & 12,3 & 9,8 & \\
\hline
\end{tabular}

(1) Os valores de concentração (total) são dados com base na matéria seca. ${ }^{(2)}$ Os valores de concentração para o N na forma amoniacal e de nitrato foram determinados na amostra nas condições originais. ${ }^{(3)}$ CMP (Concentração Máxima Permitida) P 4230, CETESB (1999).

os valores para Cu foram altos, inclusive os da testemunha. Para o $\mathrm{Zn}$, houve aumento do teor disponível no solo com a aplicação de lodo nas três profundidades. Na testemunha, o teor de $\mathrm{Zn}$ já se encontrava alto em 0-0,05 m, mas a adição de lodo nas doses $L 1$ e $L 2$ aumentou este val or em cinco e onze vezes, respectivamente. O teor matéria orgânica foi maior somente em 0-0,05 m, na dose L2. Não houve diferença estatística a $5 \%$ na CTC e na soma de bases (S).

No segundo ano, não houve diferença estatística nos teores de $\mathrm{P}$, indicando que, provavelmente, a adubação recomendada, queadicionou $21 \mathrm{~kg} \mathrm{ha}^{-1} \mathrm{de}$ $\mathrm{P}$ ao solo em cada ano no tratamento testemunha, também acumulou $\mathrm{P}$ disponível na superfície, onde todos os três tratamentos enquadraram-se na faixa considerada alta para P.

O acúmul o de metais acentuou-se no segundo ano e também aumentou em profundidade. Houve diferença significativa entre a doses $L 0$ e $L 2$ para Cu e Ni em 0-0,05 e 0,05-0,10 m e para Zn nas três profundidades. Os teores de $\mathrm{Zn}$ aumentaram nove e 18 vezes nas doses $L 1$ e $L 2$, respectivamente, em relação à dose $L 0$, na camada de $0-0,05 \mathrm{~m}$. No segundo ano houve aumento nos teores de Zn também na testemunha (L0).

Verificou-se aumento significativo na matéria orgânica somente na camada de 0-0,05 m e na CTC nas três profundidades. Na soma de bases, houve diminuição na camada de 0-0,05 m, mas não houve diferença em 0,05-0,10 e 0,10-0,20 m. No segundo ano, houve diminuição significativa no $\mathrm{pH}$ do solo com a aplicação do lodo na camada de 0-0,05 m.

O aumento no teor matéria orgânica em solo onde foi aplicado LE também foi observado por Bataglia et al. (1983a). Meloet al. (1994) encontraramaumento de carbono orgânico com aplicação de LE apenas na dose mais elevada (32 t ha-1) até 230 dias após a aplicação. Esses autores também verificaram aumento na CTC do solo nos tratamentos com LE, como no presente ensaio, atribuindo esse efeito ao aumento do carbono orgânico do solo.

O maior acúmulo de metais em forma disponível no segundo ano pode estar relacionado a três fatores: o aporte adicional de metais via segunda aplicação de lodo; a distribuição mais homogênea dos metais acumulados no solo a partir do segundo ano de aplicação, e a diminuição no pH, que sabidamente influi na disponibilidade de metais. O aumento nos teores de metais pesados em solo onde foi aplicado LE tem sido relatado em experimentos de campo e Iaboratório (Anjos \& Mattiazo, 2000; Cornu et al., 2001; M bila et al., 2001).

Em relação ao $\mathrm{pH}$, ressalta-se que a prática de adição de cal ao lodo para sua estabilização écomum. Diversos autores já observaram aumento do pH do sol o com a adi ção de lodo cal ado (Oliveira, 1995, Silva et al., 1998). No presente ensaio, no entanto, observou-se redução do pH. Destaca-se que o lodo utilizado neste experimento não foi tratado com cal, mas com polieletrólitos. Outro fator que pode ter contribuído para essa redução de $\mathrm{pH}$ é produção de áci dos orgâni cos e inorgânicos, $\mathrm{como} \mathrm{H}_{2} \mathrm{SO}_{4} \mathrm{eHNO}_{3}$, pel a decomposi ção da matéria orgânica do lodo e pela atividade microbiana. Brady (1990) descreve um experimento em que, após seis anos de aplicação de LE, observou-se acidificação do solo. E ste efeito foi também relatado por Dahlin et al. (1997), que verificaram, após 23 anos de aplicação de lodo, diminuição no pH de 6,1 para 5,8. Tsadilas et al. (1995), em estudo de vasos, observaram redução do $\mathrm{pH}$ de 7,4 para 7,1, atribuindo esta diminuição à maior nitrificação induzida pelo aporte de $\mathrm{N}$ via LE.

\section{Formas de fósforo no solo}

A fração mais solúvel de $\mathrm{P}$ (extraída por $\mathrm{CaCl}_{2}$ ) apresentou valores semel hantes para as três doses 
Quadro 3. Teores de $\mathbf{P}$ (resina), $\mathrm{Cu}, \mathrm{Ni}, \mathrm{Zn}, \mathrm{pH}\left(\mathrm{CaCl}_{2}\right)$, matéria orgânica (M.O.), soma de bases (S) e capacidade de troca catiônica (CTC) nas profundidades de 0-0,05, 0,05-0,10 e 0,10-0,20 m, após aplicação de adubo NPK (L 0) e lodo de esgoto (L 1 e L 2)

\begin{tabular}{|c|c|c|c|c|c|c|c|c|c|c|c|c|}
\hline \multirow{2}{*}{ Camada } & \multicolumn{3}{|c|}{$\mathbf{P}$} & \multicolumn{3}{|c|}{$\mathrm{Cu}$} & \multicolumn{3}{|c|}{$\mathbf{N i}$} & \multicolumn{3}{|c|}{ Zn } \\
\hline & LO & LI & $\mathbf{L 2}$ & LO & L 1 & L2 & LO & LI & $\mathbf{L 2}$ & LO & L 1 & L2 \\
\hline & \multicolumn{12}{|c|}{$\mathrm{mg} \mathrm{dm}^{-3}$} \\
\hline & \multicolumn{12}{|c|}{$2001 / 02$} \\
\hline $0-0,05$ & $31 \mathrm{~b}$ & $46 a b$ & $55 a$ & $6,3 a$ & $7,5 \mathrm{a}$ & $7,9 a$ & $0,2 \mathrm{a}$ & $0,3 a$ & $0,5 \mathrm{a}$ & $1,9 c$ & $10,0 \mathrm{~b}$ & $22,1 \mathrm{a}$ \\
\hline $0,05-0,10$ & $15 a$ & $25 a$ & $26 a$ & $6,3 a$ & 7,0 a & $7,0 \mathrm{a}$ & $0,2 \mathrm{a}$ & $0,3 a$ & $0,3 a$ & $1,1 \mathrm{c}$ & $4,7 \mathrm{~b}$ & $9,2 \mathrm{a}$ \\
\hline \multirow[t]{2}{*}{$0,10-0,20$} & $9 a$ & $11 \mathrm{a}$ & 11 a & $6,0 a$ & $6,7 \mathrm{a}$ & $6,1 \mathrm{a}$ & $0,3 a$ & $0,2 \mathrm{a}$ & $0,2 \mathrm{a}$ & $0,7 \mathrm{~b}$ & $2,1 \mathrm{ab}$ & $3,4 a$ \\
\hline & \multicolumn{12}{|c|}{$2002 / 03$} \\
\hline $0-0,05$ & $46 a$ & $47 a$ & $56 a$ & $6,4 b$ & $11,3 \mathrm{a}$ & $13,9 a$ & $0,2 c$ & $0,5 \mathrm{~b}$ & $0,9 a$ & $2,7 c$ & $24,3 \mathrm{~b}$ & $48,8 \mathrm{a}$ \\
\hline $0,05-0,10$ & 31 a & $28 \mathrm{a}$ & 33 a & $6,6 \mathrm{~b}$ & 9,0 a & $9,8 \mathrm{a}$ & $0,2 \mathrm{~b}$ & $0,4 \mathrm{~b}$ & $0,6 \mathrm{a}$ & $2,2 c$ & $14,2 \mathrm{~b}$ & $24,2 \mathrm{a}$ \\
\hline \multirow[t]{4}{*}{$0,10-0,20$} & 11 a & $14 a$ & 12 a & $6,4 a$ & $7,9 a$ & 7,8 a & $0,2 \mathrm{a}$ & $0,3 a$ & $0,4 a$ & $1,1 \mathrm{~b}$ & $5,4 a b$ & $10,0 a$ \\
\hline & \multicolumn{3}{|c|}{$\mathrm{pH}\left(\mathrm{CaCl}_{2}\right)$} & \multicolumn{3}{|c|}{ M.O. } & \multicolumn{3}{|c|}{ S } & \multicolumn{3}{|c|}{ СТС } \\
\hline & & & & 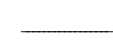 & $\mathrm{ng} \mathrm{dm} \mathrm{dm}^{-3}$ & - & 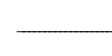 & - & $-\mathrm{mmol}$ & $\mathrm{dm}^{-3}$ & & + \\
\hline & \multicolumn{12}{|c|}{$2001 / 02$} \\
\hline $0-0,05$ & $5,6 a$ & $5,7 \mathrm{a}$ & $5,4 a$ & $26 b$ & $29 a b$ & 31 a & $71,7 \mathrm{a}$ & 97,7 a & 95,3 a & 99,3 a & $124,4 \mathrm{a}$ & $127,4 \mathrm{a}$ \\
\hline $0,05-0,10$ & $5,0 \mathrm{a}$ & 5,1 a & 5,1 a & $23 a$ & $26 a$ & $26 a$ & $38,4 a$ & 49,7 a & $51,4 a$ & 76,0 a & 88,6 a & 90,5 a \\
\hline \multirow[t]{2}{*}{$0,10-0,20$} & $4,7 a$ & $4,8 a$ & $4,8 a$ & $24 a$ & $23 a$ & $23 a$ & $27,6 a$ & $34,3 a$ & $34,1 \mathrm{a}$ & 70,0 a & 77,8 a & 75,2 a \\
\hline & \multicolumn{12}{|c|}{$2002 / 03$} \\
\hline $0-0,05$ & $5,8 \mathrm{a}$ & $5,3 \mathrm{~b}$ & $5,0 \mathrm{c}$ & $24 b$ & $28 a b$ & $32 \mathrm{a}$ & $66,2 \mathrm{a}$ & $59,3 a b$ & $52,7 b$ & $88,8 \mathrm{~b}$ & $93,4 a b$ & $103,4 a$ \\
\hline $0,05-0,10$ & 5,6 a & $5,3 a$ & $5,2 \mathrm{a}$ & $24 a$ & $26 a$ & $27 a$ & $54,0 a$ & 52,7 a & 59,4 a & $79,9 \mathrm{~b}$ & $86,4 a b$ & $99,8 \mathrm{a}$ \\
\hline $0,10-0,20$ & $4,9 a$ & $4,8 a$ & $4,7 a$ & $22 a$ & $23 a$ & $23 a$ & 33,0 a & $33,0 \mathrm{a}$ & 37,2 a & $75,4 \mathrm{~b}$ & 75,6 b & 89,6 a \\
\hline
\end{tabular}

Para cada atributo, letras diferentes, na linha, representam diferença estatística a $5 \%$ de probabilidade entre os tratamentos pelo teste t, em cada profundidade.

(Figura 1), o que era previsível, pois se passaram mais deseis meses após a aplicação de lodo, incluindo a estação de chuvas. Nestas condições, o P mais solúvel na camada de 0-0,05 m já teria sido transportado pela água de enxurrada, na forma

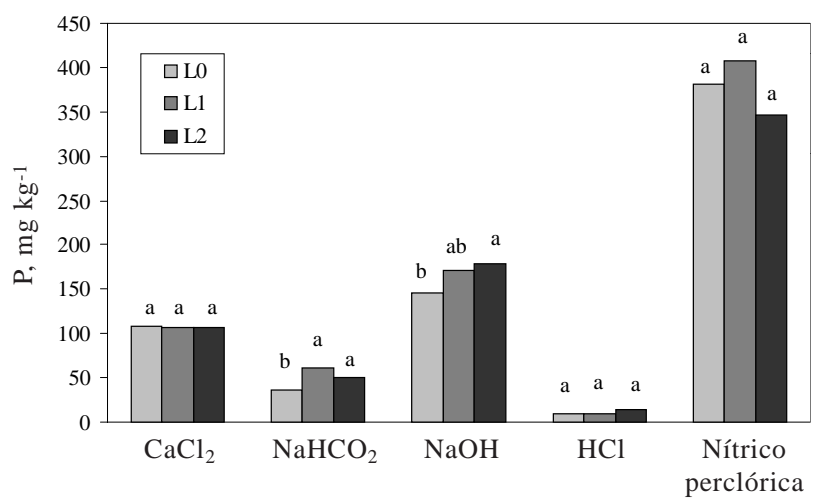

Figura 1. Extração seqüencial de $P$ em solo (00,05 m) nas doses L0, L 1, e L2, 234 dias após aplicação do lodo (primeiro ano). Letras diferentes representam difer ença estatística a $5 \%$ pelo teste " $\mathrm{t}$ ". solúvel, fixado na matéria orgânica ou em outras partículas do sol o ou absorvido pela cultura.

Houve também diferença significativa entre a dose $L 0$ e as doses L1 e L2 na quantidade de P extraído por $\mathrm{NaHCO}_{3}$ (disponível). Essa ocorrência deve estar ligada à observação descrita por Bowman $\&$ Cole(1978) de que estereagente extrai quantidade significativa de P orgânico de substratos. O P solúvel ligadoa óxidos deAl eFe (extraído com $\mathrm{NaOH}$ ) compõe a maior parte, em conformidade com a literatura. Segundo Camargo et al. (1976), os óxidos de Fe e Al são os principais responsáveis pela retenção de fosfatos em nossos sol os ácidos. Este fato explica, pel o menos em parte, a diferença significativa entre a dose $\mathrm{L} 0$ e a dose $\mathrm{L} 2$ na extração por $\mathrm{NaOH}$.

Munhoz (2001) também verificou aumento das frações mais lábeis $\left(\mathrm{CaCl}_{2}\right.$ e $\left.\mathrm{NaHCO}_{3}\right)$ e moderadamente lábeis $(\mathrm{NaOH})$ em Latossolo Vermelho distrófico que recebeu LE. I sto também foi observado no presente experimento, evidenciando o fato de que se, por um lado, a adição de lodo pode disponibilizar $P$, esta disponibilidade pode ser rapidamente reduzida pela interação do nutriente com os componentes dos solos tropicais. Sui \& Thompson (2000), ao submeterem ao fracionamento amostras de 
solo onde foi aplicado LE, concluíram que a quantidade de $\mathrm{P}$ que pode ser dessorvida do solo aumentou com a aplicação de lodo, aumentando a concentração de P solúvel em água, em bicarbonato, em hidróxidos e em HCl. Maguire et al. (2000) anal isaram sol os agrícol as que receberam aplicações de biossólido e fracionaram as amostras para P. A adição de lodo aumentou os teores de P disponível, mas também aumentou significativamente os teores de $\mathrm{P}$ ligado ao Fe e apresentou uma forte tendência de aumento na fração ligada aos óxidos de Al.

A fraçãoligada a $\mathrm{Ca}$ (extraída com $\mathrm{HCl}$ ) apresentou valores baixos e não houve diferença entre os tratamentos. E mbora tenha recebido calagem, o solo não continha al tos val ores de Ca eo lodo não recebeu adição de cal. A fração residual também não diferiu entre as doses, mas deve ser ressaltado que esta foi a fração preponderante comparada às outras.

\section{Produção de milho}

As parcel as com aplicação de lodo de esgoto ( $L 1$ e L2) apresentaram produções até $25 \%$ superiores às parcelas sem lodo e que receberam adubação com NPK (LO), embora nãotenha sido observada diferença estatística para a produção de grãos no primeiro ano (Quadro 4).

Anjos \& Mattiazzo (2000) não encontraram diferenças significativas entre a produtividade dos tratamentos com biossólido e com adubação convencional, em experimento com a cultura de milho em vasos. Por outrolado, Tsadilas et al. (1995) observaram maior produção de grãos com a aplicação de lodo de esgoto, assim como Cripps et al. (1992), que verificaram, em um ano de baixa pluviosidade, uma produtividade de grãos $47 \%$ maior com aplicação de $11,2 \mathrm{Mg} \mathrm{ha}^{-1}$ de lodo, em comparação com a adubação convencional. Silva et al. (2002) relataram maior produção de grãos em relação à testemunha absoluta e à adubação NPK por três anos, após uma única aplicação de lodo, demonstrando seu efeito residual.

\section{Teores de elementos químicos na planta}

Para o $\mathrm{P}$, embora os teores disponíveis no solo (Quadro 2) tenham sido significativamente diferentes entre os tratamentos LO e L 2 apenas na profundidade de 0-0,05 m no primeiro ano, os teores nas fol has apresentaram diferenças significativas (Quadro 5). Esse fato pode estar refletindo as diferenças encontradas para as formas de $\mathrm{P}$ no solo na camada de 0-0,05 m (Figura 1). Houve aumento significativo dos teores de $Z n$ nas fol has, refletindo o acúmulo desse elemento nas parcelas que receberam lodo, nos dois anos avaliados. O Ni não foi determinado nas folhas no primeiro ano. No segundo ano, não houve diferença entre os tratamentos para os teores de $\mathrm{Ni}$ nas fol has, embora o solo apresentasse diferença significativa entre as três doses na camada de $0-0,05$ m e entre L 0 e L 2 na camada de 0,05-0,10 m. Os teores foliares de Cu apresentaram diferença significativa entre os tratamentos L 0 e L 2 no primeiro ano, mas não houve diferença entre os tratamentos no segundo ano.

\section{CONSIDERAÇÕES FINAIS}

A preocupação com o acúmulo de $\mathrm{P}$ no solo não se justificou pelos dados obtidos. Os teores de $\mathrm{P}$ disponíveis no solo onde foi aplicado lodo foram semel hantes aos do tratamento com adubo NPK. Entretanto, a aplicação do lodo aumentou as frações lábeis e moderadamente lábeis do $\mathrm{P}$ na camada superficial. Segundo Munhoz (2001), esse P adsorvido fracamente poderia estar sendo levado por

Quadro 5. Teores médios de P, Cu, Ni e Zn nas folhas de milho nos tratamentos sem aplicação de lodo e com adubação NPK, LO, e lodo de esgoto, L 1 e L2, nos anos agrícolas de 2001/2002 e 2002/2003

\begin{tabular}{|c|c|c|c|c|c|c|c|}
\hline \multirow{2}{*}{ Dose } & \multicolumn{3}{|c|}{$2001 / 02$} & \multicolumn{4}{|c|}{$2002 / 03$} \\
\hline & $\mathbf{P}$ & Zn & $\mathrm{Cu}$ & $\mathbf{P}$ & Zn & $\mathrm{Cu}$ & $\mathbf{N i}$ \\
\hline & $\mathrm{g} \mathrm{kg}^{-1}$ & $-\mathrm{mg}$ & $\mathrm{kg}^{-1}$ & $\mathrm{~g} \mathrm{~kg}^{-1}$ & 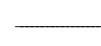 & $-\mathrm{g} \mathrm{kg}^{-1}$ & + \\
\hline LO & $2,35 \mathrm{a}$ & $12,3 \mathrm{a}$ & $9,3 \mathrm{a}$ & $2,8 \mathrm{a}$ & 37,0 a & $12,0 \mathrm{a}$ & $2,8 \mathrm{a}$ \\
\hline L1 & $2,67 \mathrm{~b}$ & $23,0 \mathrm{~b}$ & 9,9 ak & $3,1 \mathrm{~b}$ & $48,5 \mathrm{~b}$ & $12,0 \mathrm{a}$ & $2,6 a$ \\
\hline L2 & $2,72 \mathrm{~b}$ & $36,0 \mathrm{c}$ & 10,4 b & $3,2 \mathrm{~b}$ & $71,7 \mathrm{c}$ & $12,6 \mathrm{a}$ & $4,0 \mathrm{a}$ \\
\hline
\end{tabular}

Para cada variável, letras diferentes, na linha, representam diferença estatística a $5 \%$ entre os tratamentos, pelo teste " $t$ ".

Quadro 4. Valores médios da produção de matéria seca e de grãos de milho, considerando as doses de lodo empregadas (LO, L 1 e L2)

\begin{tabular}{|c|c|c|c|c|c|c|}
\hline \multirow{2}{*}{ Safra } & \multicolumn{3}{|c|}{ Matéria seca } & \multicolumn{3}{|c|}{ Grão } \\
\hline & LO & LI & L2 & LO & L1 & L2 \\
\hline & & & & & 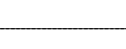 & 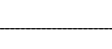 \\
\hline 2001/02 & $4.897 \mathrm{~b}$ & $5.380 \mathrm{ab}$ & $6.803 \mathrm{a}$ & $3.830 \mathrm{a}$ & $4.381 \mathrm{a}$ & $4.518 a$ \\
\hline 2002/03 & $4.713 \mathrm{~b}$ & $5.546 \mathrm{ab}$ & $5.981 \mathrm{a}$ & $4.020 \mathrm{~b}$ & $4.896 \mathrm{a}$ & $4.992 \mathrm{a}$ \\
\hline
\end{tabular}

Para cada variável, letras diferentes, na linha, representam diferença estatística a $5 \%$ entre os tratamentos, pelo teste "t". 
águas superficiais, sendo fonte de poluição para cursos de água. Esse aumento das formas mais lábeis refletiu-se em maiores teores de $P$ nas folhas de milho no segundo ano. Vale ainda lembrar queo aporte total de P via lodo não foi excessivo, considerando as características dos solos tropicais altamente intemperizados, como o solo deste experimento.

Por outro lado, houve aumento dos teores de $\mathrm{Ni}$ e Zn no solo, o que se refletiu na absorção desses elementos pela planta. Dessa forma, faz-se necessário monitoramento constante onde o lodo de esgoto for aplicado para que haja controle adequado dos teores de metais no solo.

\section{CONCLUSÕES}

1. A aplicação de lodo de esgoto em sol o cultivado com milho aumentou a produção da cultura no segundo ano, comparativamente à do tratamento sem aplicação de lodo e com adubação química.

2. Não houve efeito das doses de lodo sobre os teores de $\mathrm{P}$ no solo, mas houve incremento dos teores foliares do nutriente.

3. Verificou-se aumento dos teores de metais no solo (Cu, Ni e Zn) e na planta (Zn).

\section{LITE RATURA CITADA}

ABREU, M.F.; ABREU, C.A. \& ANDRADE , J .C. Determinação de fósforo, potássio, cálcio, magnésio, enxofre, cobre, ferro, manganês, zinco, níquel, cádmio, cromo e chumbo em ácido nítrico usando métodos de US-EPA. In: RAIJ , B. van; ANDRADE, J.C.; CANTARELLA, H. \& QUAGGIO, J .A., eds. Análise química para avaliação da fertilidade de solos tropicais. Campinas, Instituto Agronômico de Campinas, 2001. p.151-161.

ANJ OS, A.R.M. \& MATTIAZZO, M.E. Lixiviação de íons inorgânicos em solos repetidamente tratados com biossólido. R. Bras. Ci. Solo, 24:927-938, 2000.

BATAGLIA, O.C.; BERTON, R.S.; CAMARGO, O.A. \& VALADARES, J .M.A.S. Resíduos orgânicos como fontes de nitrogênio para capim-braquiária. R. Bras. Ci. Solo, 7:277-284, 1983a.

BATAGLIA, O.C.; FURLANI, A.M.C.; TEIXEIRA, J .P.F.; FURLANI,J .R. \& GALLO,J .R. Métodos deanálisequímica de plantas. Campinas, Instituto Agronômico de Campinas, 1983b. 48p. (Boletim Técnico, 78)

BERTON, R.S.; VALADARES, J .M.A.S.; CAMARGO, O.A. \& BATAGLIA, O.C. Peletização de lodo de esgoto e adição de $\mathrm{CaCO}_{3}$ na produção de matéria seca e absorção de $\mathrm{Zn}$, Cu e $\mathrm{Ni}$ pelo milho em três Latossolos. R. Bras. Ci. Solo, 21:685691, 1997.
BOWMAN, R.A. \& COLE, C.V. Transformations of organic phosphorous substrates in soils as evaluated by $\mathrm{NaHCO}_{3}$ extraction. Soil Sci., 125:49-54, 1978.

BRADY, N.C. The nature and properties of soils. 10. ed. New York, Macmillan, 1990. 621p.

CAMARGO , O.A.; RAIJ , B. van \& GROHMANN, F. Influência de cátions trocáveis e pH na fixação do fósforo em solos. Ci Cult., 28:678-681, 1976.

CANTARELLA, H. \& TRIVELIN, P.C.O. Determinação de nitrogênio total em solo. In: RAIJ , B. van; ANDRADE, J .C.; CANTARELLA, H. \& QUAGGIO, J .A., eds. Análisequímica para avaliação da fertilidade de sol os tropicais. Campinas, Instituto Agronômico de Campinas, 2001. 285p.

COMPANHIA DE TECNOLOGIA DE SANEAMENTO AMBIENTAL - CETESB. Aplicação de biossólidos de sistemas de tratamento biológico em áreas agrícolas Critérios para projeto e operação: manual técnico. São Paulo, 1999. 33p.

CORNU, S.; NEAL, C.; AMBROSI, J .P.; WHITEHEAD, P.; NEAL, M.; SIGOLO, J \& \& VACHIER, P. Theenvironmental impact of heavy metals from sewage sludge in Ferrasols (São Paulo, Brazil). Sci. Total Environ., 271:27-48, 2001.

CRIPPS, R.W.; WINFREE, S.K. \& REAGAN, J.L. Effects of sewage sludge application method on corn production. Comm. Soil. Sci. Plant Anal., 23:1705-1715, 1992.

CRITCHFIELD, H.J. General climatology. Englewood Cliffs, Prentice-Hall, 1960. 465p.

DAHLIN, S.; WITTER, E.; MART, A.; TURNER, A. \& BAATH, $E$. Where's the limit? Changes in the microbiological properties of agricultural soils at low levels of metal contamination. Soil Biol. Biochem., 29:1405-1415, 1997.

ELLIOT, H.A. Land application of municipal sewage sludge. J . Soil Water Conserv., 41:5-10, 1986.

GIBBS, R.A., HU, C.J .; HO, G.E. \& UNKOVICH, I. Regrowth of faecal coliforms and salmonellae in stored biosolids and soil amended with biosolids. Water Sci. Technol., 35:269275, 1997.

HARRIS-PIERCE, R.L.; REDENTE, E.F. \& BARBARICK, K.A. Sewage sludge application effects on runoff water quality in a semiarid grassland. J. Environ. Qual., 24:112-115, 1995.

HEDLEY, M.J .; STEWART, J.W.B. \& CHAUHUAN, B.S. Changes in inorganic and organic soil phosphorus fractions induced by cultivation practices and laboratory incubations. Soil Sci. Soc. Am. J ., 46:970-976, 1982.

J ORGE, J.A.; CAMARGO, O.A. \& VALADARES, J.M.A.S. Condições físicas de um $L$ atossolo Vermel ho-Escuro, quatro anos após a aplicação de lodo de esgoto e cal cário. R. Bras. Ci. Solo, 15:237-240, 1991.

MAGUIRE, R.O.; SIMS, J.T. \& COALE, F.J. Phosphorus fractionation in biosolids-amended soils: relationship to soluble and desorbable phosphorus. Soil Sci. Soc. Am. J., 64:2018-2024, 2000. 
MBILA, M.O.; MICHAEL, L.T.; MBAGWU, J.S.C. \& LAIRD, D.A. Distribution and movement of sludge-derived trace metals in selected Nigerian soils. J . Environ. Qual., 30:16671674, 2001.

MELO, W.J .; MARQUES, M.O.; SANTIAGO, G.; CHELLI, R.A. \& LEITE, S.A.S. Efeito de doses crescentes de lodo de esgoto sobre frações da matéria orgânica e CTC de um Latossolo cultivado com cana-de-açúcar. R. Bras. Ci. Solo, 18:449-455, 1994.

MUNHOZ, R.C. Disponibilidade de fósforo para o milho em solo que recebeu lodo de esgoto. Campinas, I nstituto Agronômico de Campinas, 2001. 74p. (Tese de Mestrado)

OLIVEIRA, F.C. Metais pesados eformas nitrogenadas em solos tratados com lodo de esgoto. Piracicaba, Escola Superior de Agricultura "Luis deQueiroz", 1995. 90p. (Tese de Mestrado)

POLGLASE, P.J . \& MYERS, B.J . Tree plantations for recycling effluent and biosolids in Australia. In: ELDRIDGE, K.G., ed. Environmental management: the role of eucalypts and other fast cropping species: proceedings. Collingwood, CSIRSO, 1996. p.100-109.

RAIJ , B. van; ANDRADE, J .C.; CANTARELLA, H. \& QUAGGIO, J .A., eds. Análise química para avaliação da fertilidade de solos tropicais. Campinas, Instituto Agronômico de Campinas, 2001. 285p.

SILVA, F.C.; BOARETTO, A.E.; BERTON, R.S.; ZOTELLI, H.B.; PEIXE, C.A. \& MENDONÇA, E. Cana-de-açúcar cultivada em solo adubado com lodo de esgoto: nutrientes, metais pesados e produtividade. Pesq. Agropec. Bras., 33:1-8, 1998.
SILVA, J .E.; RESCK, D.V.S. \& SHARMA, R.D. Alternativa Agronômica para o biossólido produzido no distrito federal. I - Efeito na produção de milho e na adição de metais pesados em Latossolo no cerrado. R. Bras. Ci. Solo, 26:487495, 2002.

SUI, Y. \& THOMPSON, M.L. Phosphorus sorption, desorption, and buffering capacity in a biosolids-amended mollisol. Soil Sci. Soc. Am. J ., 64:164-169, 2000.

SUI, Y.; THOMPSON, M.L. \& SHANG, C. Fractionation of phosphorus in a Mollisol amended with biosolids. Soil Sci. Soc.Am. J ., 63:1174-1180, 1999.

TAN, K.H. Environmental soil science. 2.ed. Nova York, Marcel Dekker, 2000. 480p.

TSADILAS, C.D.; MATSI, T.; BARBAYIANNIS, N. \& DIMOYIANNIS, D. Influence of sewage sludge application on soil properties and on the distribution and availability of heavy metal fractions. Comm. Soil.Sci. Plant. Anal., 26:2603-2619, 1995.

TSUTIYA, M.T. Alternativas de disposição final de biossólidos. In:TSUTIYA, M.T.; COMPARINI, J .B.; SOBRINHO, P.A.; HESPANHOL, I.; CARVALHO, P.C.T.; MELFI, A.J .; MELO, W.J . \& MARQUES, M.O., eds. Biossólidos na agricultura. São Paulo, SABESP, 2001. 468p.

WILSON, S.C.; DUARTE-DAVIDSON, R. Z\& J ONES, K.C. Screening the environment fate of organic contaminants in sewage sludges applied to agricultural soil: 1 . The potential for downward movement to groundwaters. Sci. Total Environ., 185:45-57, 1996. 